\title{
Cause of plasmasphere corotation lag
}

\author{
J. L. Burch and J. Goldstein \\ Southwest Research Institute, San Antonio, Texas, USA \\ B. R. Sandel \\ Lunar and Planetary Laboratory, University of Arizona, Tucson, Arizona, USA
}

Received 25 November 2003; revised 28 January 2004; accepted 6 February 2004; published 3 March 2004.

[1] It is hypothesized that the recently observed plasmasphere corotation lag is caused by a corresponding corotation lag in the upper ionosphere. Rotation rates of long-lived plasmaspheric notches are compared to ionospheric drifts observed on DMSP spacecraft in the same longitude sectors. Good agreement between the two observations is found, and the cause of the corotation lag is identified as the ionospheric disturbance dynamo. The observed corotation lag will have to be accounted for in future magnetospheric convection models, which until now have assumed strict plasmasphere corotation. INDEX TERMS: 2712 Magnetospheric Physics: Electric fields (2411); 2730 Magnetospheric Physics: Magnetosphere-inner; 2736 Magnetospheric Physics: Magnetosphere/ionosphere interactions; 2760 Magnetospheric Physics: Plasma convection; 2768 Magnetospheric Physics: Plasmasphere. Citation: Burch, J. L., J. Goldstein, and B. R. Sandel (2004), Cause of plasmasphere corotation lag, Geophys. Res. Lett., 31, L05802, doi:10.1029/ 2003 GL019164.

\section{Introduction}

[2] Sandel et al. [2003] used localized low-density regions (or notches) as markers to track the rotation of the plasmasphere. Such notches have been observed to persist for periods as long as 60 hours, during which time they can be tracked for up to six hours of each 14-hour IMAGE [Burch, 2000] orbit when the spacecraft is hovering near apogee. Sandel et al. found that the plasmasphere rotation rate at $\mathrm{L} \sim 2-3$ lags corotation by up to $10-15 \%$. Although no cause of the corotation lag was given, its observation has strong implications for magnetospheric convection models [e.g., Grebowsky, 1970; Spiro et al., 1981; McIlwain, 1986; Weiss et al., 1997], which have for many years assumed a corotation electric field inside a "shielding distance" at which the Region-2 field-aligned current system shields the inner magnetosphere from the electric fields produced by the interaction of the magnetosphere with the solar wind. It is worth noting that the corotation lag observed by Sandel et al. [2003] is not caused by the simple penetration of outer magnetospheric electric fields into the plasmasphere, although such effects surely occur, because it does not show a strong local-time dependence. Penetration fields would produce a generally sunward flow that at these $\mathrm{L}$ values would cause a higher rotation rate in the dawn hemisphere and a lower rate in the dusk hemisphere.
[3] The large corotation lags in Jupiter's magnetosphere are attributable either to plasma mass loading in the Io plasma torus [Pontius and Hill, 1982; Brown, 1994] or to the subsequent outward transport of that plasma [Hill, 1979, 1980; McNutt et al., 1979]. However, application of the same mechanism to Earth [Hill, 1979] fails by orders of magnitude to explain a corotation lag of $\sim 10-15 \%$ at $\mathrm{L} \sim$ $2-3$ as observed by Sandel et al. [2003]. The IMAGE observations, which show a percentage corotation lag at $2-$ 3 Earth radii two to three times that in the Io plasma torus (even though there is no local gas source as there is at Io), suggest a strong influence but one that is not related to mass loading. The simplest conclusion, and the hypothesis upon which this study was based, is that a departure from corotation of the midlatitude ionosphere is the direct cause of plasmasphere subcorotation. Ionospheric corotation is generally assumed to hold in plasmaspheric convection models; however, radar and satellite observations [e.g., Wand, 1983; Heelis and Coley, 1992] have for many years shown that a significant departure from corotation, especially at midlatitudes, does routinely occur. Furthermore, its cause has been suggested to be the ionospheric disturbance dynamo [Blanc and Richmond, 1980]. In addition to an ionospheric corotation lag, the upper atmosphere and ionosphere at times exhibit superrotation [Rishbeth, 1972]. Whether corotation, subcorotation, or superrotation occurs at any given place and time depends upon the confluence of numerous effects including, but not limited to, angular momentum conservation, solar tidal forces, auroral heating, and ionospheric currents.

[4] Two data sources are used in this study - EUV images of the plasmasphere from the IMAGE satellite and ion drift measurements from the DMSP F12, F13 and F15 satellites. These data, applied to one of the events published by Sandel et al. [2003], demonstrate that subcorotation of the ionosphere is fully consistent with the observed subcorotation of the plasmasphere. The implications of this result are important for the development of accurate models of magnetospheric convection electric fields, which must include either real-time empirical data or accurate models of global ionospheric drifts if accurate descriptions of inner magnetosphere convection are to be obtained. For example, a $10-15 \%$ corotation lag will cause the boundary between open and closed convection paths to move closer to the Earth than would be predicted by a model involving strict plasmaspheric corotation.

\section{Observations}

[5] Figure 1 shows a sequence of plasmasphere images obtained on April 6-8, 2001 by the EUV instrument 


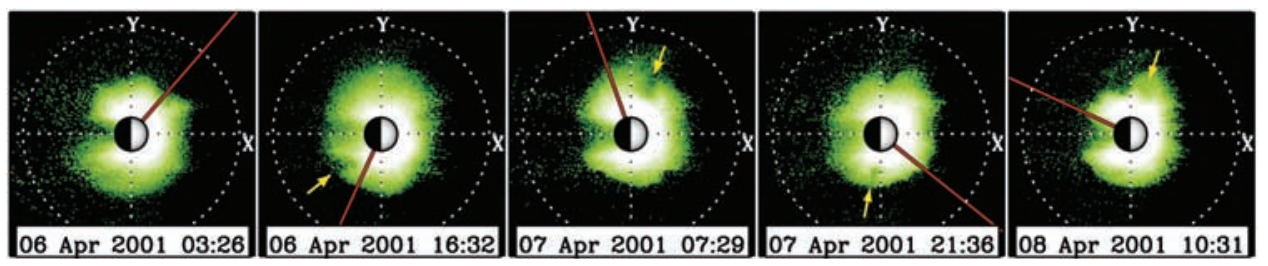

Figure 1. EUV images in 30.4-nm light plotted in L-MLT on April 6-8, 2001. Each frame of EUV data is an accumulation of 30.4-nm light from five two-minute spin periods of the IMAGE satellite. Each observed pixel of columnintegrated EUV irradiance is mapped to the equator along the dipole field line corresponding to the minimum-L intersection of its field of view [Goldstein et al., 2003]. The red radial line, which bisects a plasmaspheric density notch in the first panel, tracks the Earth's rotation in the subsequent panels in which the notch is noted with a yellow arrow.

[Sandel et al., 2000] on the IMAGE satellite. In each panel is an EUV image that has been mapped to the magnetic equator in L and magnetic local time (MLT) [see Roelof and Skinner, 2000; Goldstein et al., 2003b; Dent et al., 2003]. For the line of sight corresponding to the center of each pixel, the mapping procedure identifies the dipole field line of minimum $\mathrm{L}$ value touched by the line of sight and assigns the brightness of the pixel to the [L, MLT] coordinates of that field line. This procedure is based on the fact that the plasmaspheric density falls off rapidly with $\mathrm{L}\left(\sim \mathrm{L}^{-4}\right)$ and is most accurate where sharp edges occur in the density profile (e.g., at the plasmapause). The color scale intensity is proportional to the log of the line-of-sight integrated $\mathrm{He}^{+}$column abundance. In the center of each image, which is a view from above the north pole, the apparent size and location of the Earth are indicated by the black and white circle. The Sun is to the right in each panel, and a faint shadow extends antisunward from the Earth on the nightside. The plasmasphere is the greenish-to-white haze of 30.4-nm light that surrounds the Earth; close to the Earth the emissions are dominated by airglow from neutral helium and $\mathrm{O}^{+}$.

[6] In each panel of Figure 1 a red line is drawn radially outward from the Earth, and in the first panel the line bisects a plasmaspheric density notch. In subsequent panels the red line moves with the rotation of the Earth, while the notch, which is marked with a yellow arrow, is clearly seen to lag significantly behind corotation over the 55-hour period covered by the five panels.

[7] In order to test the hypothesis that the corotation lag of plasmaspheric notch features follows the overall plasmasphere and ionosphere rotation rate, drift-meter data from the DMSP spacecraft F12, F13 and F15 were compiled for the magnetic longitude sector $100^{\circ}-180^{\circ}$. This range of longitudes bounds the location of the notch throughout its period of observation beginning on April 6, 2001. These data are plotted in Figure 2 with the six-hour MLT sectors color coded. Also shown in each panel are the Kp values during the respective ten-hour periods. Positive values of the ordinate correspond to eastward drift, which is defined relative to the $96^{\circ}$ orbital plane of DMSP so that only drift meter data, and not RPA data, are used. The rationale for this restriction is the same as that used by Heelis and Coley [1992]. The drift meter data are obtained with higher time resolution, are affected to a lesser degree than the RPA data by sources of error such as spacecraft potential variations, and for the midlatitude region occupied by the outer plasmasphere are very close to representing geographic or magnetic east-west flow velocities.
[8] Each panel in Figure 2 covers a ten-hour time period beginning with the first observation of the plasmaspheric notch early on April 6, 2001. In each panel, high and variable values of east-west drift associated with the auroral oval are clearly seen at latitudes above $50^{\circ}-60^{\circ}$. At low and midlatitudes, the drifts are generally within $100 \mathrm{~m} / \mathrm{s}$ of the corotation velocity, which is denoted by the 0-line in Figure 2; but since the corotation velocity at the DMSP altitude of $\sim 850 \mathrm{~km}$ and at $45^{\circ}$ magnetic latitude is only $\sim 325 \mathrm{~m} / \mathrm{s}$, the variations are quite significant. As shown by the color-coded MLT sectors, there are significant dependencies on MLT and latitude, and these features of the data in Figure 2 are consistent with those published by Heelis and Coley [1992]. For example, a supercorotation at midnight for magnetic latitudes near $25^{\circ}$ and a subcorotation at all MLTs remote from noon at midlatitudes are clearly seen in Figure 2 as well as in Figure 3 of Heelis and Coley [1992].

[9] In Figure 3, the observed location of the plasmaspheric density notch in UT-MLT is plotted by the red data points. The position of a corotating feature, beginning at the first observation of the notch at 03:03 UT on April 6, 2001, is shown by the blue line. Finally, the predicted locations of the notch after each of the first five ten-hour periods of notch observation (the first five panels of Figure 2) are shown by the green data points, which are derived from the average east-west drift velocity at latitudes between $40^{\circ}$ and $45^{\circ}$. This latitude range represents that of the Earthward edge of the notch. Extension of the drift data averages to higher latitudes yields comparable results but with larger errors because of contamination by auroral drifts.

[10] The notch locations plotted in Figure 3 were derived visually from the projected EUV images (in Figure 1) by selecting the innermost point of each notch at the average MLT covered by it. These identifications were checked by an independent automated procedure, which involved brightness profiles taken around an annulus within the EUV images. The annulus covered $\mathrm{L}$ values between 2.4 and 3.1. For the five images shown in Figure 1, the locations derived by the two techniques differed by an average of $3.5^{\circ}$ or 0.23 hours in MLT. This error in notch locations, if it occurs randomly, would lead to a $\sim 1 \%$ error in rotational period. A more rigorous statistical analysis, using the full set of measurements in Figure 3 and the formalism described by Bevington [1969] (p. 114) confirms this estimate, yielding an angular velocity of the notch of $92 \pm 1 \%$ of corotation. Using all the measurements in 

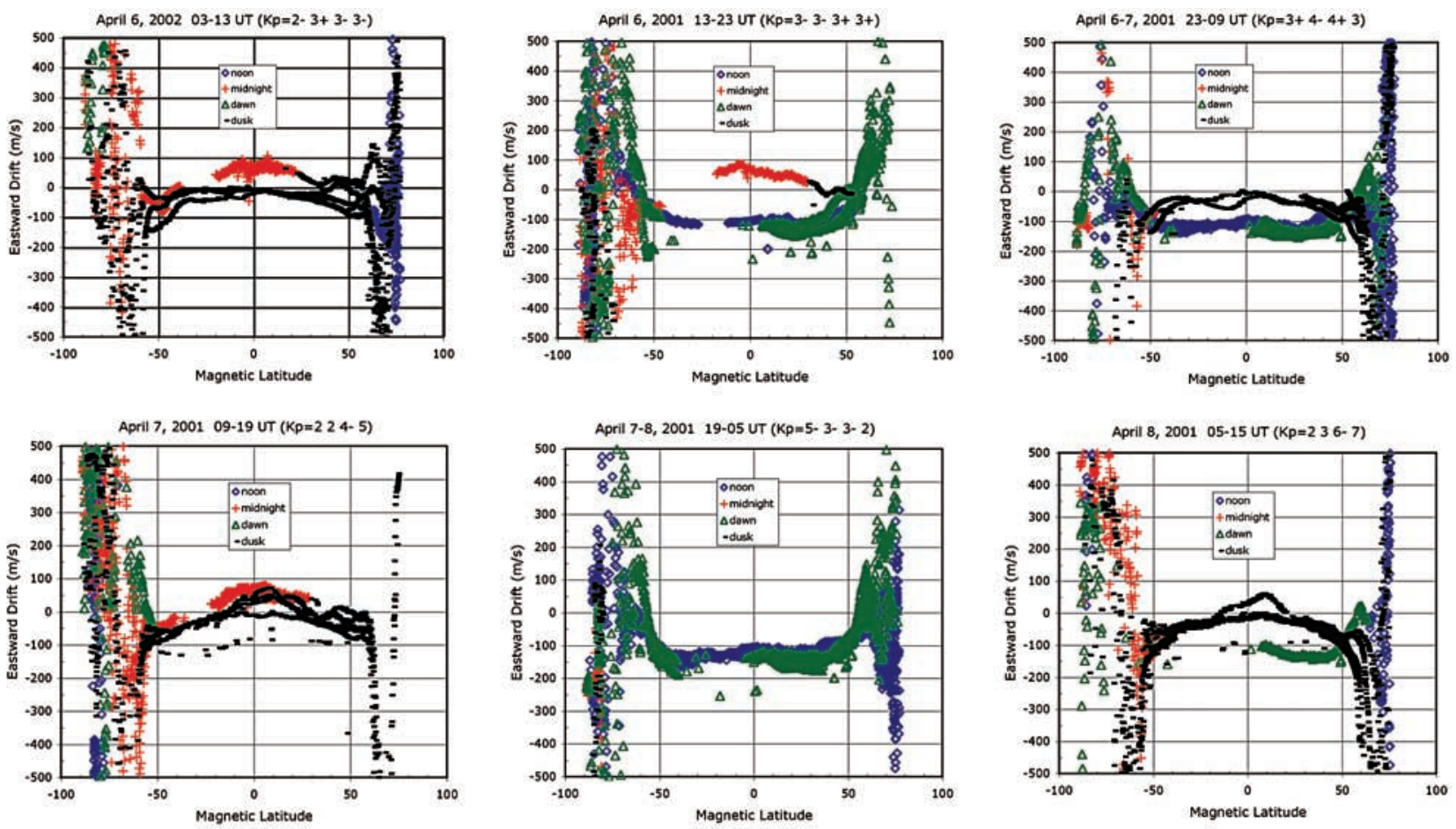

Figure 2. Drift-meter data from the DMSP 12, 13 and 15 satellites for six ten-hour periods during the observation of the plasmaspheric notch shown in Figure 1. Each plot is a composite of data from all three satellites whenever they were between geomagnetic longitudes of $100^{\circ}$ and $180^{\circ}$, which defines the longitude sector containing the notch throughout its period of observation. Noon (09-15 UT), midnight (21-03 UT), dawn (03-09 UT) and dusk (15-21 UT) data are color coded. Kp values for the ten-hour period are listed at the top of each plot.

Figure 7c of Sandel et al. [2003], we find a consistent overall value of $90 \pm 1 \%$ of corotation.

\section{Discussion and Conclusions}

[11] The data shown in Figures 1-3 show clearly that the outer plasmasphere, as traced by a density notch feature, rotates at a rate significantly slower $(\sim 10 \%)$ than corotation, as first noted by Sandel et al. [2003]. Analysis of DMSP data for the same spatial and temporal locations occupied by the notch show that the departures from corotation observed in the midlatitude upper ionosphere are fully capable of predicting the plasmaspheric corotation lag when the MHD approximation is assumed to hold for the region above $850 \mathrm{~km}$ altitude. Thus, while the observed corotation lag should not be surprising, it is considerably at odds with the usual assumption of strict corotation that has been used in all magnetospheric convection models that have been in use for at least the past three decades.

[12] The cause of the corotation lag at midlatitudes is most likely the ionospheric disturbance dynamo as described by Blanc and Richmond [1980]. In its simplest terms, this phenomenon involves the input of energy to the auroral ionosphere by particle precipitation and joule heating. This heat input produces equatorward winds that carry gas into the midlatitude region where the rotational velocity of the Earth is increasing. Conservation of angular momentum within this gas (or equivalently the Coriolis force), which originated at higher latitudes, causes it to lag behind the Earth's rotation. This lag at latitudes just below the auroral oval is clearly seen in the DMSP data of Figure 2.
[13] The most obvious result of a slower plasmasphere rotation rate in magnetospheric convection models is that the boundary between open and closed convection paths will move closer to the Earth. In addition, the details of the convection patterns throughout the inner magnetosphere will be affected since at every point the corotation electric

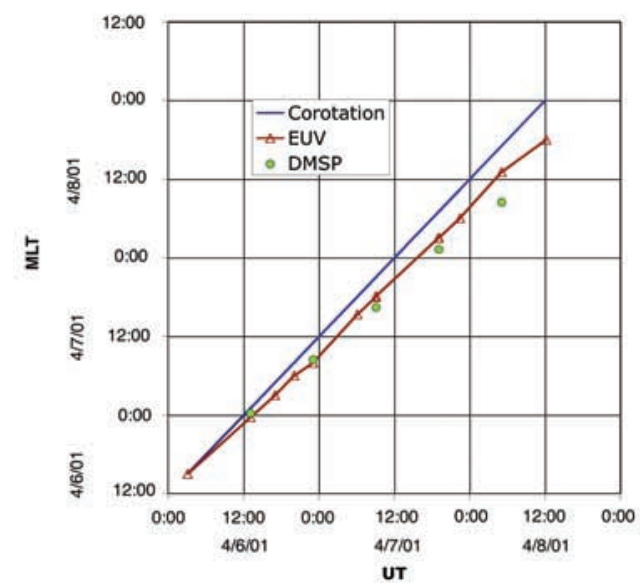

Figure 3. Plot in MLT and UT of position of the plasmaspheric notch from its first observation at 03:05 UT on April 6, 2001 until 12:03 UT on April 8, 2001 (red data points); the locations predicted by corotation (blue line), and the locations predicted by the DMSP data in Figure 2 within the geomagnetic latitude interval $40^{\circ}-45^{\circ}$ (green data points). 
field and any externally imposed electric fields are added to produce a total electric field.

[14] One can estimate the effects of the corotation lag by considering the analytical Volland-Stern electric potential model $\left(\Phi=\Phi_{\mathrm{C}} / \mathrm{L}+\Phi_{\mathrm{M}} \mathrm{L}^{2} \sin (\varphi)\right)$ [Volland, 1973; Stern, 1975] where $\Phi_{C}$ and $\Phi_{M}$ are the corotation potential and the magnetospheric convection potential, respectively, and $\varphi$ is an azimuthal angle measured counterclockwise from midnight. The location of the last closed equipotential (LCE) in the equatorial dusk meridian $\left(\varphi=270^{\circ}\right.$, where both the corotation electric field and the magnetospheric electric field are aligned radially) can be obtained by setting $\mathrm{E}$ (the sum of the corotation electric field, $E_{C}$, and the magnetospheric electric field, $\mathrm{E}_{\mathrm{M}}$ ) equal to zero, i.e.,

$$
\begin{aligned}
& \mathrm{E}_{\left(\varphi=270^{\circ}\right)}=-\partial \Phi_{(\varphi=270)} / \partial \mathrm{L}=-\Phi_{\mathrm{C}} \mathrm{L}^{-2}+2 \Phi_{\mathrm{M}} \mathrm{L}=0, \text { or } \\
& \mathrm{L}^{3}=\Phi_{\mathrm{C}} /\left(2 \Phi_{\mathrm{M}}\right) .
\end{aligned}
$$

Thus, for a given value of the magnetospheric potential, the L-value of the dusk-meridian LCE in the equatorial plane varies as the cube root of the corotation potential. Maynard and Chen [1975] have derived a specific Kp dependence of $\Phi_{\mathrm{M}}$, which can be used to show, for example, that for $\mathrm{Kp}=4$ the equatorial dusk meridian LCE will lie at $\mathrm{L}=5.2$ for strict corotation but at $\mathrm{L}=4.9$ for a $15 \%$ corotation lag applied uniformly throughout the plasmasphere.

[15] It is interesting to note that, although the Hill [1979] model does not apply to Earth, there is a point of commonality in the physical mechanisms at work. In both cases, the corotation lag results from the Coriolis force acting on plasma that is being transported "outward" (away from the spin axis). At Jupiter, the outward plasma transport occurs in the equatorial plane and results from the centrifugal instability of the Iogenic plasma. At Earth, the "outward" plasma transport occurs in the ionosphere and results from the disturbance dynamo effect. (T. W. Hill, personal communication, 2003).

[16] Since the observed corotation lag is significant, it is important that future convection electric-field models treat this phenomenon accurately either by incorporating empirical ionospheric data or by including a realistic model of midlatitude ionospheric convection that reflects the systematic variations with latitude and local time (as illustrated in Figure 2 and previously presented by Heelis and Coley [1992]) that are known to occur. In fact, the latitude dependence shown in Figure 2 suggests that the plasmaspheric rotation speed varies significantly with radial distance.

[17] Acknowledgments. This research was supported by NASA Contract No. NAS5-96020 with Southwest Research Institute. A helpful discussion with Dr. Don Carpenter is gratefully acknowledged. Cynthia Farmer provided significant assistance with data reduction. The DMSP drift-meter data were provided by the PI, Dr. R. A. Heelis, through the UTDallas DMSP web site. Helpful comments by both referees are appreciated.

\section{References}

Bevington, P. R. (1969), Data Reduction and Error Analysis for the Physical Sciences, McGraw Hill, New York.

Blanc, M., and A. D. Richmond (1980), The ionospheric disturbance dynamo, J. Geophys. Res., 85, 1669-1686.

Brown, M. E. (1994), Observation of mass loading in the Io plasma torus, Geophys. Res. Lett., 21, 847-850.

Burch, J. L. (2000), IMAGE mission overview, Space Sci. Rev, 91, 1-14. Dent, Z. C., I. R. Mann, F. W. Menk, J. Goldstein, C. R. Wilford, M. A. Clilverd, and L. G. Ozeke (2003), A coordinated ground-based and IMAGE satellite study of quiet-time plasmaspheric density profiles, Geophys. Res. Lett., 30(12), 1600, doi:10.1029/2003GL016946.

Goldstein, J., M. Spasojevic, P. H. Reiff, B. R. Sandel, W. T. Forrester, D. L. Gallagher, and B. W. Reinisch (2003), Identifying the plasmapause in IMAGE EUV data using IMAGE RPI in situ steep density gradients, J. Geophys. Res., 108(A4), 1147, doi:10.1029/2002JA009475.

Grebowsky, J. M. (1970), Model study of plasmapause motion, J. Geophys. Res., 75, 4329-4333.

Heelis, R. A., and R. J. Coley (1992), East-west ion drifts at mid-latitudes observed by Dynamics Explorer 2, J. Geophys. Res., 97, 19,461-19,469.

Hill, T. W. (1979), Inertial limit on corotation, J. Geophys. Res., 84, 65546558.

Hill, T. W. (1980), Corotation lag in Jupiter's magnetosphere: Comparison of observation and theory, Science, 207, 301-302.

Maynard, N. C., and A. J. Chen (1975), Isolated cold plasma regions: Observations and their relation to possible production mechanisms, J. Geophys. Res., 80, 1009-1013.

McIlwain, C. E. (1986), A Kp dependent equatorial electric field model, Adv. Space Res., 6, 187-197.

McNutt, R. L., Jr., J. W. Belcher, J. D. Sullivan, F. Bagenal, and H. S. Bridge (1979), Departure from rigid corotation of plasma in Jupiter's dayside magnetosphere, Nature, 280, 803.

Pontius, D. H., and T. W. Hill (1982), Departure from corotation of the Io plasma torus: Local plasma production, Geophys. Res. Lett., 9, 13211324.

Rishbeth, H. (1972), Superrotation of the upper atmosphere, Rev. Geophys., 10, 799-819.

Roelof, E. C., and A. J. Skinner (2000), Extraction of ion distributions from magnetospheric ENA and EUV images, Space Sci. Rev., 91, 437-459.

Sandel, B. R., et al. (2000), The extreme ultraviolet (EUV) imager investigation for the IMAGE mission, Space Sci. Rev., 91, 197-242.

Sandel, B. R., J. Goldstein, D. L. Gallagher, and M. Spasojevic (2003), EUV observations of the structure and dynamics of the plasmasphere, Space Sci. Rev., 109, 25-46.

Spiro, R. W., M. Harel, R. A. Wolf, and P. H. Reiff (1981), Quantitative simulation of a magnetospheric substorm: 3. Plasmaspheric electric fields and evolution of the plasmapause, J. Geophys. Res., 86, 2261-2272.

Stern, D. P. (1975), The motion of a proton in the equatorial magnetosphere, J. Geophys. Res., 80, 595-599.

Volland, H. (1973), A semiempirical model of large-scale magnetospheric electric fields, J. Geophys. Res., 78, 171-180.

Wand, R. H. (1983), Seasonal variations of lower thermospheric winds from the Millstone Hill incoherent scatter radar, J. Geophys. Res., 88, 9227-9241.

Weiss, L. A., R. L. Lambour, R. C. Elphic, and M. F. Thomsen (1997), Study of plasmaspheric evolution using geosynchronous observations and global modeling, Geophys. Res. Lett., 24, 599-602.

J. L. Burch and J. Goldstein, Southwest Research Institute, P. O. Drawer 28510, San Antonio, TX 78228-0510, USA. (jburch@swri.edu; jgoldstein@swri.edu)

B. R. Sandel, Lunar and Planetary Laboratory, University of Arizona, 901 Gould Simpson Bldg., Tucson, AZ 85721, USA. (sandel@arizona.edu) 